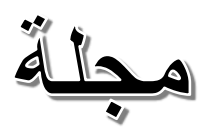

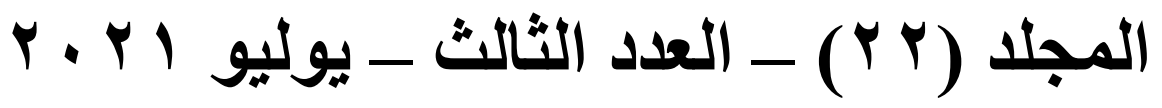

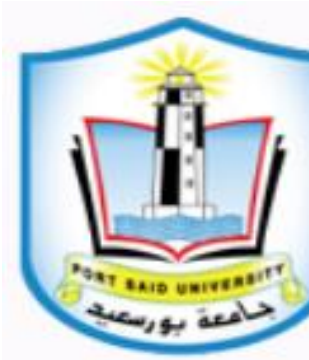

\title{
The exponentiated Akash distribution with properties and applications
}

\section{Pro. Nasr Ibrahim Rashwan}

Professor and Head of the Department of Statistics, Mathematics and Insurance, Faculty of Commerce, Tanta University

\section{Dr.Abdelreheem Awad Bassuny}

Phd in statistics, Faculty of Commerce, Tanta University 


\begin{abstract}
In this study, a new two-parameter lifetime distribution named the exponentiated Akash distribution is introduced and studied. Akash distribution is considered an extension of the lindley distribution and can be easily expressed as a mixture of an exponential distribution with scale parameter $(\theta)$ and a gamma distribution with shape parameter equal 3 and scale parameter $\theta$. The reliability (survival) function, hazard rate function (also known as the failure rate function), the reversed hazard rate and the cumulative hazard rate function of the new distribution are obtained. Some statistical properties of the exponentiated Akash distribution such as the moments, moment generating function, order statistics, Renyi entropy and quantile function are derived. The method of maximum likelihood is used to estimate the unknown parameters of this distribution. Finally, the performance of this new proposed distribution is examined using some real life data sets to show its flexibility and better goodness of fit as compared with other lifetime distributions such as Akash and exponential based on the Akaike information criterion.
\end{abstract}

Keywords: exponentiated Akash distribution, moment generating function, order statistics, Renyi entropy, Maximum Likelihood estimation 


\section{Introduction}

The Akash distribution is proposed by Shanker (2015) as a new oneparameter lifetime distribution. This distribution can be easily expressed as a mixture of an exponential distribution with scale parameter $(\theta)$ and a gamma distribution with shape parameter equal 3 and scale parameter $\theta$ as follows:

$$
g(x)=p g_{1}(x)+(1-p) g_{2}(x)
$$

Where $p=\frac{\theta^{2}}{\theta^{2}+2}$ and $1-p=\frac{2}{\theta^{2}+2}$ are mixing proportions, $g_{1}(x)=\theta e^{-\theta x}$ is the probability density function (pdf) of exponential distribution and $g_{2}(x)=\frac{\theta^{3} x^{2} e^{-\theta x}}{2}$ is the pdf of gamma distribution. The pdf and cdf of Akash distribution are given respectively as:

and

$$
g(x)=\frac{\theta^{3}}{\theta^{2}+2}\left(1+x^{2}\right) e^{-\theta x} ; x>0, \quad \theta>0
$$

$$
G(x)=1-\left(1+\frac{\theta x(\theta x+2}{\theta^{2}+2}\right) e^{-\theta x} ; x>0, \quad \theta>0
$$

The exponentiated distribution is derived by raising the cumulative density function of an arbitrary parent distribution to an additional positive parameter (Gupta et al (1998)). Let X denote a random variable from an arbitrary parent distribution, the cdf of the resulting exponentiated is given by

$$
F(x)=[G(x)]^{\alpha}, \quad \alpha>0
$$

Where $G(x)$ is the cdf of the parent distribution. The corresponding pdf is obtained by differentiating eq. (4) with respect to $\mathrm{x}$ as follows:

$$
f(x)=\alpha[G(x)]^{\alpha-1} g(x)
$$

The idea of exponentiated distribution was suggested by Gupta et al (1998), who discussed a new family has two- parameter (Scale and Shape). Some mathematical properties of the exponentiated exponential are derived by Gupta and Kundu (2001).

In a similar manner, Nadarajah (2005) introduced the exponentiated Gumbel distribution. Pal et al (2006) discussed the exponentiated weibull distribution. Nadarajah and Gupta (2007) proposed the exponentiated gamma distribution. Showky and Abu-Zinadah (2009) investigated the 
exponentiated pareto distribution. Lemonte and Cordeiro (2011) presented the exponentiated generalized inverse Gaussian distribution Ashour and Eltehiwy (2015) proposed the exponentiated power lindley distribution. Elgarhy and showki (2017) studied the exponentiated Sushila distribution. Jabeen and Para (2018) introduced the exponentiated gamma exponential distribution and Uwaeme et al (2018) proposed an extended Pranav distribution.

This paper offers a new distribution named exponentiated Akash (EA) distribution. This article is organized as follows: In section 2, The EA distribution is proposed and defined. Some statistical properties of this distribution are derived and discussed in section 3. Section 4 involves maximum likelihood estimation method. Section 5 provides application to real data sets. Finally, some conclusions are presented in section 6 .

\section{Exponentiated Akash distribution}

In this section, we propose and introduce the two-parameter exponentiated Akash (EA) distribution. Based on eq. (3) in eq. (4), the cdf of the EA distribution is given by

$$
F(x)=\left[1-\left(1+\frac{\theta x(\theta x+2)}{\theta^{2}+2}\right) e^{-\theta x}\right]^{\alpha} ; x>0, \quad \theta, \alpha>0
$$

and the corresponding pdf of this distribution is defined as

$$
f(x)=\frac{\alpha \theta^{3}}{\theta^{2}+2}\left(1+x^{2}\right) e^{-\theta x}\left[1-\left(1+\frac{\theta x(\theta x+2)}{\theta^{2}+2}\right) e^{-\theta x}\right]^{\alpha-1} ; x>0, \quad \theta, \alpha>0
$$

where $\theta$ is scale parameter and $\alpha$ is shape parameter.The reliability (survival) function, $\mathrm{R}(\mathrm{S})$, hazard rate function (also known as the failure rate function), $h(x)$, the reversed hazard rate, $\mathrm{r}(\mathrm{x})$ and the cumulative hazard rate function, $(\mathrm{H}(\mathrm{x})$ of the EA distribution are given by:

$$
\begin{gathered}
R(x)=1-F(x)=1-\left[1-\left(1+\frac{\theta x(\theta x+2)}{\theta^{2}+2}\right) e^{-\theta x}\right]^{\alpha} \\
h(x)=\frac{f(x)}{R(x)}=\frac{\alpha\left(\frac{\theta^{3}}{\theta^{2}+2}\right)\left(1+x^{2}\right) e^{-\theta x}\left[1-\left(1+\frac{\theta x(\theta x+2)}{\theta^{2}+2}\right) e^{-\theta x}\right]^{\alpha-1}}{1-\left[1-\left(1+\frac{\theta x(\theta x+2)}{\theta^{2}+2}\right) e^{-\theta x}\right]^{\alpha}} \\
r(x)=\frac{f(x)}{F(x)}=\frac{\alpha \theta^{3}\left(1+x^{2}\right) e^{-\theta x}}{\left(\theta^{2}+2\right)\left[1-\left(1+\frac{\theta x(\theta x+2)}{\theta^{2}+2}\right) e^{-\theta x}\right]}
\end{gathered}
$$

and 


$$
H(x)=-\ln R(x)=-\ln \left\{1-\left[1-\left(1+\frac{\theta x(\theta x+2)}{\theta^{2}+2}\right) e^{-\theta x}\right]^{\alpha}\right\}
$$

Note that, if $\alpha=1$, then the EA distribution in eq. (7) reduces to the Akash distribution in eq. (2) with parameter $\theta$.

Here, simple expansions for the cdf and pdf of the EA distribution will be derived as follows

Using the following generalized binomial theorem if $\mathrm{b}$ is positive and $|z|<$ 1 , then

$$
(1-Z)^{b-1}=\sum_{i=0}^{\infty}(-1)^{i}\left(\begin{array}{c}
b-1 \\
i
\end{array}\right) Z^{i} \quad ;|Z|<1
$$

The cdf of the EA distribution in eq. (6) can be rewritten as

let

$$
F(x)=\sum_{i, j=0}^{\infty} \sum_{k=0}^{j}(-1)^{i}\left(\begin{array}{c}
\alpha \\
i
\end{array}\right)\left(\begin{array}{l}
i \\
j
\end{array}\right)\left(\begin{array}{l}
j \\
k
\end{array}\right) \frac{2^{k} \theta^{2 j-k}}{\left(\theta^{2}+2\right)^{j}} x^{2 j-k} e^{-i \theta x}
$$

$$
S_{i j k}=\sum_{i, j=0}^{\infty} \sum_{k=0}^{j}(-1)^{i}\left(\begin{array}{l}
\alpha \\
i
\end{array}\right)\left(\begin{array}{l}
i \\
j
\end{array}\right)\left(\begin{array}{l}
j \\
k
\end{array}\right) \frac{2^{k} \theta^{2 j-k}}{\left(\theta^{2}+2\right)^{j}}
$$

then

$$
F(x)=S_{i j k} x^{2 j-k} e^{-i \theta x} ; \quad x>0, \quad \theta, \alpha>0
$$

and the pdf of the EAdistribution is given by

$$
f(x)=\sum_{i, j=0}^{\infty} \sum_{k=0}^{j}(-1)^{i}\left(\begin{array}{c}
\alpha-1 \\
i
\end{array}\right)\left(\begin{array}{l}
i \\
j
\end{array}\right)\left(\begin{array}{l}
j \\
k
\end{array}\right) \frac{2^{k} \alpha \theta^{2 j-k+3}}{\left(\theta^{2}+2\right)^{j+1}}\left(x^{2 j-k}+x^{2 j-k+2}\right) e^{-\theta x(i+1)}
$$

let

then

$$
W_{i j k}=\sum_{i, j=0}^{\infty} \sum_{k=0}^{j}(-1)^{i}\left(\begin{array}{c}
\alpha-1 \\
i
\end{array}\right)\left(\begin{array}{l}
i \\
j
\end{array}\right)\left(\begin{array}{l}
j \\
k
\end{array}\right) \frac{2^{k} \alpha \theta^{2 j-k+3}}{\left(\theta^{2}+2\right)^{j+1}}
$$

$$
f(x)=W_{i j k}\left(X^{2 j-k}+X^{2 j-k+2}\right) e^{-\theta x(i+1)}
$$

\section{Statistical properties of the EA distribution}

\subsection{The moments}

The $\mathrm{r}^{\text {th }}$ moment of a random variable $\mathrm{X}$ having the EA distribution with parameters $\theta$ and $\alpha$ can be derived. Using eq. (10), the $\mathrm{r}^{\text {th }}$ moment is given by 


$$
E\left(X^{r}\right)=\grave{\mu_{r}}=W_{i j k} \int_{0}^{\infty} X^{r}\left(X^{2 j-k}+X^{2 j-k+2}\right) e^{-\theta x(i+1)} d x
$$

Based on the transformation $y=\theta x(i+1)$, then $y>0, X=\frac{y}{\theta(i+1)}$ and $d x=\frac{d y}{\theta(i+1)}$

$$
\begin{aligned}
E\left(X^{r}\right)=\grave{\mu_{r}} & =W_{i j k} \int_{0}^{\infty}\left[\left(\frac{y}{\theta(i+1)}\right)^{r+2 j-k}+\left(\frac{y}{\theta(i+1)}\right)^{r+2 j-k+2}\right] e^{-y} \frac{d y}{\theta(i+1)} \\
& =W_{i j k}\left[\frac{\Gamma(r+2 j-k+1)}{(\theta(i+1))^{r+2 j-k+1}}+\frac{\Gamma(r+2 j-k+3)}{(\theta(i+1))^{r+2 j-k+3}}\right]
\end{aligned}
$$

Where $\Gamma($.$) is the gamma function$

So, the first four moments of the EA distribution are given by

$$
\begin{aligned}
& \grave{\mu_{1}}=W_{i j k}\left[\frac{\Gamma(2 j-k+2)}{(\theta(i+1))^{2 j-k+2}}+\frac{\Gamma(2 j-k+4)}{(\theta(i+1))^{2 j-k+4}}\right], \\
& \grave{\mu_{2}}=W_{i j k}\left[\frac{\Gamma(2 j-k+3)}{(\theta(i+1))^{2 j-k+3}}+\frac{\Gamma(2 j-k+5)}{(\theta(i+1))^{2 j-k+5}}\right], \\
& \grave{\mu_{3}}=W_{i j k}\left[\frac{\Gamma(2 j-k+4)}{(\theta(i+1))^{2 j-k+4}}+\frac{\Gamma(2 j-k+6)}{(\theta(i+1))^{2 j-k+6}}\right], \\
& \grave{\mu_{4}}=W_{i j k}\left[\frac{\Gamma(2 j-k+5)}{(\theta(i+1))^{2 j-k+5}}+\frac{\Gamma(2 j-k+7)}{(\theta(i+1))^{2 j-k+7}}\right],
\end{aligned}
$$

Based on the first four moments, the measures of mean $(\mu)$, the variance $\left(\sigma^{2}\right)$, skewness coefficient $(\mathrm{SK})$, and Kurtosis coefficient $(\mathrm{Ku})$ can be calculated as

and

$$
\begin{aligned}
& \mu=\grave{\mu_{1}}=W_{i j k}\left[\frac{\Gamma(2 j-k+2)}{(\theta(i+1))^{2 j-k+2}}+\frac{\Gamma(2 j-k+4)}{(\theta(i+1))^{2 j-k+4}}\right] \\
& \sigma^{2}=\grave{\mu_{2}}-{\grave{\mu_{1}}}^{2} \quad, S K=\frac{\grave{\mu_{3}}-3 \grave{\mu_{1} \dot{\mu}_{2}}+2{\grave{\mu_{1}}}^{3}}{\left(\grave{\mu_{2}}-{\grave{\mu_{2}}}^{2}\right)^{3 / 2}}
\end{aligned}
$$

\subsection{Moment generating function}

Let $X$ have as exponentiated Akash distribution, then the moment generating function of $X,(M g f)$, say $M_{X}(t)$ is given by

$$
M_{X}(t)=E\left(e^{t x}\right)=W_{i j k} \int_{0}^{\infty}\left(X^{2 j-k}+X^{2 j-k+2}\right) e^{-X(\theta(i+1)-t)} d x
$$


By using the transformation $y=X[\theta(i+1)-t]$, we get

$$
M_{X}(t)=W_{i j k}\left[\frac{\Gamma(2 j-k+1)}{(\theta(i+1)-t)^{2 j-k+1}}+\frac{\Gamma(2 j-k+3)}{(\theta(i+1)-t)^{2 j-k+3}}\right]
$$

In the same way, the factorial moment generating function, $M_{X}(\ln t)$ and the characteristic function $\phi_{x}(t)$ of this distribution are given by

$$
M_{X}(\ln t)=W_{i j k}\left[\frac{\Gamma(2 j-k+1)}{(\theta(i+1)-\ln t)^{2 j-k+1}}+\frac{\Gamma(2 j-k+3)}{(\theta(i+1)-\ln t)^{2 j-k+3}}\right]
$$

and

$$
\phi_{x}(t)=W_{i j k}\left[\frac{\Gamma(2 j-k+1)}{(\theta(i+1)-i t)^{2 j-k+1}}+\frac{\Gamma(2 j-k+3)}{(\theta(i+1)-i t)^{2 j-k+3}}\right]
$$

Where $i=\sqrt{-1}$ is the unit imaginary

\subsection{Distribution of order statistics}

Suppose $X_{1}, X_{2}, \ldots, X_{n}$ is a random sample of size $\mathrm{n}$ from EA distribution. Let $X_{(1)}<X_{(2)}<X_{(3)}<\cdots<X_{(n)}$ denote the corresponding order statistics. The pdf and cdf of the $\mathrm{i}^{\text {th }}$ order statistic, cay, $f_{i: n}(x)$ and $F_{i: n}(x)$ for $i=1,2, \ldots, n$ are given by

$$
f_{i: n}(x)=\frac{n ! f(x)}{(i-1) !(n-i) !}[F(x)]^{i-1}[1-F(x)]^{n-i}
$$

Using the binomial series expansion, we get

and

$$
\begin{aligned}
& f_{i: n}(x)= \frac{n ! f(x)}{(i-1) !(n-i) !} \sum_{j=0}^{\infty}(-1)^{j}\left(\begin{array}{c}
n-i \\
j
\end{array}\right)[F(x)]^{i+j-1} \\
&=\sum_{j, m, k=0}^{\infty} \sum_{p=0}^{k}(-1)^{j+m}\left(\begin{array}{c}
n-i \\
j
\end{array}\right)\left(\begin{array}{c}
\alpha(i+j)-1 \\
m
\end{array}\right)\left(\begin{array}{c}
m \\
k
\end{array}\right)\left(\begin{array}{l}
k \\
p
\end{array}\right) \\
& \\
& \times \frac{2^{p} \alpha \theta^{2 k-p+3}}{\left(\theta^{2}+2\right)^{k+2}}\left(x^{2 k-p}+x^{2 k-p+2}\right) e^{-\theta x(k+1)}
\end{aligned}
$$

$$
\begin{aligned}
F_{i: n}(x) & =\sum_{j=i}^{n}\left(\begin{array}{l}
n \\
j
\end{array}\right)(F(x))^{j}(1-F(x))^{n-j} \\
& =\sum_{j=i}^{n} \sum_{k=0}^{n-j}(-1)^{k}\left(\begin{array}{c}
n \\
j
\end{array}\right)\left(\begin{array}{c}
n-j \\
k
\end{array}\right)(F(x))^{j+k} \\
& =\sum_{j=i}^{n} \sum_{k=0}^{n-j}(-1)^{k}\left(\begin{array}{c}
n \\
j
\end{array}\right)\left(\begin{array}{c}
n-j \\
k
\end{array}\right)\left[1-\left(1+\frac{\theta x(\theta x+2)}{\theta^{2}+2}\right) e^{-\theta x}\right]^{j+k}
\end{aligned}
$$

\subsection{Renyi Entropy}


Entropy of a random variable $\mathrm{X}$ is a measure of variation of uncertainty. One of the type of entropy widely used is Renyi entropy (Renyi (1961). If $\mathrm{X}$ is a continuous random variable having EA distribution, then the Renyi entropy is defined as

$$
\mathrm{I}_{\mathrm{x}: \mathrm{R}}(\mathrm{q})=\frac{1}{1-\mathrm{q}} \ln \left(\mathrm{I}_{\mathrm{x}}(\mathrm{q})\right)
$$

Where $\mathrm{I}_{\mathrm{x}}(\mathrm{q})=\int_{\mathrm{R}} \mathrm{f}^{\mathrm{q}}(\mathrm{x}) \mathrm{dx} \quad, \mathrm{q}>0$ and $q \neq 1$

$$
\begin{aligned}
& \mathrm{I}_{\mathrm{x}}(\mathrm{q})=\left(\frac{\alpha \theta^{3}}{\theta^{2}+2}\right)^{q} \int_{0}^{\infty} e^{-\theta q x}\left[1-\left(1+\frac{\theta x(\theta x+2)}{\theta^{2}+2}\right) e^{-\theta x}\right]^{q(\alpha-1)} d x \\
&+\left(\frac{\alpha \theta^{3}}{\theta^{2}+2}\right)^{q} \int_{0}^{\infty} x^{2 q} e^{-\theta q x}\left[1-\left(1+\frac{\theta x(\theta x+2)}{\theta^{2}+2}\right) e^{-\theta x}\right]^{q(\alpha-1)} d x
\end{aligned}
$$

Using the binomial series expansion, then

$$
\begin{gathered}
{\left[1-\left(1+\frac{\theta x(\theta x+2)}{\theta^{2}+2}\right) e^{-\theta x}\right]^{q(\alpha-1)}=\sum_{i, j=0}^{\infty} \sum_{k=0}^{j}(-1)^{i}\left(\begin{array}{c}
q(\alpha-1) \\
i
\end{array}\right)\left(\begin{array}{l}
i \\
j
\end{array}\right)\left(\begin{array}{l}
j \\
k
\end{array}\right)} \\
\times \frac{2^{k} \theta^{2 j-k} x^{2 j-k}}{\left(\theta^{2}+2\right)^{j}} e^{-\theta j x} \\
\mathrm{I}_{\mathrm{x}}(\mathrm{q})=\sum_{i, j=0}^{\infty} \sum_{k=0}^{j}(-1)^{i}\left(\begin{array}{c}
q(\alpha-1) \\
i
\end{array}\right)\left(\begin{array}{l}
i \\
j
\end{array}\right)\left(\begin{array}{l}
j \\
k
\end{array}\right) \frac{2^{k} \alpha^{q} \theta^{3 q+2 j-k}}{\left(\theta^{2}+2\right)^{q+j}} \int_{0}^{\infty}\left[x^{2 j-k}\right. \\
\left.+x^{2 q+2 j-k}\right] e^{-\theta x(q+j)} d x
\end{gathered}
$$

Let $\mathrm{A}_{i j k}=\sum_{i, j=0}^{\infty} \sum_{k=0}^{j}(-1)^{i}\left(\begin{array}{c}q(\alpha-1) \\ i\end{array}\right)\left(\begin{array}{l}i \\ j\end{array}\right)\left(\begin{array}{l}j \\ k\end{array}\right) \frac{2^{k} \alpha^{q} \theta^{3 q+2 j-k}}{\left(\theta^{2}+2\right)^{q+j}}$

By using the transformation $y=\theta x(q+j)$, then

Hence, the Renyi entropy becomes

$$
\mathrm{I}_{\mathrm{x}}(\mathrm{q})=\mathrm{A}_{i j k}\left[\frac{\Gamma(2 j-k+1)}{(\theta(q+j))^{2 j-k+1}}+\frac{\Gamma(2 q+2 j-k+1)}{(\theta(q+j))^{2 q+2 j-k+1}}\right]
$$

$$
\mathrm{I}_{\mathrm{X}: \mathrm{R}}(\mathrm{q})=\frac{1}{1-q} \ln \left[\mathrm{A}_{i j k}\left(\frac{\Gamma(2 j-k+1)}{(\theta(q+j))^{2 j-k+1}}+\frac{\Gamma(2 q+2 j-k+1)}{(\theta(q+j))^{2 q+2 j-k+1}}\right)\right]
$$

\subsection{Quantile function}

The quantile function, say $\mathrm{Q}(\mathrm{U})=\mathrm{F}^{-1}(\mathrm{U})$ of the EA distribution can be computed by inverting eq.(6) as follows

$$
\left(1+\frac{\theta x_{q}\left(\theta x_{q}+2\right)}{\theta^{2}+2}\right) e^{-\theta x_{q}}=1-u^{\frac{1}{\alpha}}
$$

We can easily generate $\mathrm{X}$ variable by taking $\mathrm{U}$ as a uniform variable in $(0,1)$

\section{Maximum likelihood estimation}


Let $x_{1}, x_{2}, \ldots, x_{n}$ be an independent random sample of size $\mathrm{n}$ from the EA distribution with parameters $\theta$ and $\alpha$, the $\log$-likelihood function, $\ln L$, can be obtained as

$$
\begin{aligned}
& \ln L=n \ln \alpha+3 n \ln \theta-n \ln \left(\theta^{2}+2\right)+\sum_{i=1}^{n} \ln \left(1+x_{i}^{2}\right)-\theta \sum_{i=1}^{n} x_{i} \\
&+(\alpha-1) \sum_{i=1}^{n} \ln \left[1-\left(1+\frac{\theta x_{i}\left(\theta x_{i}+2\right)}{\theta^{2}+2}\right) e^{-\theta x_{i}}\right]
\end{aligned}
$$

In order to maximize the log-likelihood, we solve the nonlinear equations obtained from the differentiation of eq.(19) with respect to $\theta$ and $\alpha$ as follows

$$
\begin{aligned}
\frac{\partial \ln L}{\partial \theta}=\frac{3 n}{\theta}- & \frac{2 n \theta}{\theta^{2}+2} \\
& -\sum_{i=1}^{n} x_{i} \theta^{2}(\alpha-1) \sum_{i=1}^{n} \frac{x_{i} e^{-\theta x_{i}}\left[\frac{\left(1+x_{i}^{2}\right)}{\left(\theta^{2}+2\right)}\right]}{\left[1-\left(1+\frac{\theta x_{i}\left(\theta x_{i}+2\right)}{\theta^{2}+2}\right) e^{-\theta x_{i}}\right]}
\end{aligned}
$$

and

$$
\frac{\partial \ln L}{\partial \alpha}=\frac{n}{\alpha}+\sum_{i=1}^{n} \ln \left[1-\left(1+\frac{\theta x_{i}\left(\theta x_{i}+2\right)}{\theta^{2}+2}\right) e^{-\theta x_{i}}\right]
$$

We can obtain the estimates of the unknown parameters by setting these above nonlinear equations (20) and (21) to zero and solve them simultaneously. These equations are imimplicit form, so these may be solved by using numerical iteration such as Newton Raphson algorithm.

\section{Applications}

This section contains an application of the exponentiated Akash distribution on two examples of real data sets and compares its goodness of fit with Akash (A) and exponential (E) distributions. The first set of data represents the lifetime's data associated with relief times (in minutes) of 20 patients receiving an analgesic (Gross and Clark, 1975). The data are 1.1, $1.4,1.3,1.7,1.9,1.8,1.6,2.2,1.7,2.7,4.1,1.8,1.5,1.2,1.4,3,1.7,2.3$, 1.6, 2. The second data set is the strength data of glass of the aircraft windows (Fuller et al 1994). The data are as follows: 18.83, 20.8, 21.657, 23.03, 23.23, 24.05, 24.321, 25.5, 25.52, 25.8,26.69, 26.77, 26.78, 27.05, $27.67,29.9,31.11,33.2,33.73,33.76,33.89,34.76,35.75,35.91,36.98$, $37.08,37.09,39.58,44.045,45.29,45.381$. The required numerical evaluations are implemented using Mathcad package software. In order to compare distributions, the Akaike information criterion (AIC) statistic has been computed as follows 


$$
A I C=-2 \ln L+2 q
$$

Where $\ln L$ the $\log$-likelihood function and $\mathrm{q}$ is is the number of parameters. Table (1) gives maximum likelihood estimates $\left(\mathrm{MLE}_{\mathrm{s}}\right)$ of the model parameters for ES, A and E and the value of AIC statistic for the two real data sets.

Table (1): MLE $E_{\mathrm{s}}$ and AIC of the fitted distributions of two data sets

\begin{tabular}{|c|c|c|c|c|}
\hline \multirow{2}{*}{ Data sets } & \multirow{2}{*}{ Model } & \multicolumn{2}{|c|}{ Parameters estimate } & \multirow{2}{*}{ AIC } \\
\cline { 3 - 4 } & & $\boldsymbol{\theta}$ & $\boldsymbol{\alpha}$ & \\
\hline \multirow{3}{*}{1} & AD & $\mathbf{1 . 1 5 7}$ & - & 62 \\
& ED & $\mathbf{0 . 5 2 6}$ & - & 67.9 \\
& EAD & $\mathbf{2 . 0 3 4}$ & 2.11 & 60.3 \\
\hline \multirow{3}{*}{2} & AD & $\mathbf{0 . 0 9 7}$ & - & 243 \\
& ED & $\mathbf{0 . 0 3 2}$ & - & $\mathbf{2 7 6 . 7}$ \\
& EAD & 1.80 & 1.076 & 238.2 \\
\hline
\end{tabular}

From table (1), the best distribution corresponds to the smaller value of AIC statistic, so the exponentiated Akash (EA) distribution is better than the Akash (A) and exponential (E) for these two real data sets.

\section{Conclusions}

The article introduces a new two -parameter lifetime distribution named, Exponentiated Akash distribution, its mathematical properties such as moments, moment generating function, order statistics Renyi entropy and quantile function are derived and discussed. To estimate the unknown parameters of the new distribution, the method of maximum likelihood estimation was used used. The proposed new distribution was applied to two real data sets; the EA distribution provides a better fit than Akash and exponential distributions.

\section{References:}

1. Ashour, S.K., and Eltehiwy, M.A., (2015) exponentiated power lindley distribution, Journal of advanced research, vol. 6, pp. 895-905.

2. Elgarhy., M. and Showki, A.W., (2017), exponentiated Sushila distribution, International Journal of scientific engineering and science, vol.1, pp. 9-12.

3. Fuller, E.J., Frieman, S., Quinn, J., Quinn, G. and Carter, W., (1994), Facture mechanics approach to the design of glass aircraft windows: A case study, SPIE proc 2286, pp. 419-430.

4. Gross, A. J., and Clark, V. A., (1975), survival distributions, reliability applications in the Biometrical science, John Wily, New York.

5. Gupta, R. C., Gupta, R. D. and Gupta, P. L., (1998), modeling failure time data by Lehman alternatives, communications in statistics-theory and methods, vol. 27, pp. 887-904. 
6. Gupta, R. D. and Kundu, D. (2001), exponentiated exponential family: An alternative to gamma and weibull, Biometrical Journal, vol. 43, pp. 117130.

7. Jabeen, S. and Para, B., (2018), exponentiated gamma exponential distribution, Sohag Journal of mathematics, vol. 5, pp. 79-84.

8. Lemonte, A. J., and Cordeiro, G. M., (2011), The exponentiated inverse Gaussian distribution, statistics and probability letters, vol. 81, pp. 506517.

9. Nadarajah, S., (2005), The exponentiated Gumbel distribution with climate application, environmetrics, vol. 17, pp. 13-23.

10. Nadarajah, S., and Gupta, A. K., (2007), The exponentiated gamma distribution with applications to drought data, Calcutta statistical Association Bulletin, vol. 59, pp. 29-54.

11.Pal, M., Ali, M. M., and Woo, J. (2006), exponentiated weibull distribution, Statistica, anno.IXVI, n.2, pp.139-147.

12. Renyi, A., (1961), on measures of entropy and information, in proceedings of the $4^{\text {th }}$ Berkely symposium on mathematical statistics and probability, pp. 547-561.

13. Shanker, R., (5015), Akash distribution and its applications, International Journal of Probability and statistics, vol. 4, pp. 65-75.

14. Shawky, A., I, and Abu-Zinahah, H., (2009), exponentiated pareto distribution: different methods of estimation. Int. J. Contemp. Math. Science, vol. 4, pp. 677-693.

15. Uwaeme, O.R., Akpan, N.p., and Orumie, U.C., (2018), An extended Pranav distribution, Asian Journal of probability and statistics, vol. 2, pp. $1-15$. 